\title{
Insulin alleviates mitochondrial oxidative stress involving upregulation of superoxide dismutase 2 and uncoupling protein 2 in septic acute kidney injury
}

\author{
GUANG-DAO CHEN ${ }^{1,2}$, JUN-LIANG ZHANG ${ }^{1,3}$, YI-TING CHEN ${ }^{1}$, \\ JU-XING ZHANG ${ }^{1}$, TAO WANG ${ }^{1}$ and QI-YI ZENG ${ }^{1}$ \\ ${ }^{1}$ Center of Pediatrics, Zhujiang Hospital, Southern Medical University, Guangzhou, Guangdong 510280; \\ ${ }^{2}$ Department of Pediatrics, Central Hospital of Panyu District, Guangzhou, Guangdong 511400; ${ }^{3}$ Department of \\ Neonatology, Nanfang Hospital, Southern Medical University, Guangzhou, Guangdong 510515, P.R. China
}

Received October 31, 2017; Accepted February 19, 2018

DOI: $10.3892 /$ etm.2018.5890

\begin{abstract}
The aim of the present study was to explore the effects and mechanisms of insulin on mitochondrial oxidative stress in septic acute kidney injury (AKI). Male Sprague Dawley rats were divided randomly into four groups: Control group, sham surgery group, cecal ligation and puncture (CLP) group, and CLP plus insulin group. Blood specimens and kidney tissues were obtained at 12 and $24 \mathrm{~h}$ after surgery as separate experiments. Analyses of histology and indicators of renal injury [blood urea nitrogen (BUN) and serum creatinine (CRE) and neutrophil gelatinase-associated lipocalin (NGAL)], mitochondrial function [adenosine triphosphate (ATP) and mitochondrial membrane potential (MMP)], oxidative stress [inducible nitric oxide synthase (iNOS), reactive oxygen species (ROS) and nitric oxide (NO)], endogenous antioxidant systems [superoxide dismutase (SOD) and glutathione (GSH)] as well as the expression of uncoupling protein (UCP), PINK1 protein (a major mediator of mitophagy), PGC1 $\alpha$ protein (a major regulator of mitochondrial biogenesis) were performed. Compared with CLP group, the CLP plus insulin group had milder histological damage, higher levels of ATP and MMP as well as lower levels of BUN, serum CRE and NGAL, intrarenal iNOS, mitochondrial ROS and total NO. Moreover, the CLP plus insulin group demonstrated increased expression of SOD2 and UCP2. In contrast, insulin administration suppressed mitophagy meanwhile did not upregulate total GSH and induce mitochondrial biogenesis following CLP. These findings indicated that the upregulation of SOD2 and
\end{abstract}

Correspondence to: Professor Qi-Yi Zeng, Center of Pediatrics, Zhujiang Hospital, Southern Medical University, 253 Gongye Road, Guangzhou, Guangdong 510280, P.R. China

E-mail: zqy_88@163.com

Key words: insulin, sepsis, acute kidney injury, mitochondrial dysfunction, oxidative stress, endogenous antioxidant system, uncoupling protein 2 , mitophagy, mitochondrial biogenesis
UCP2 may be involved in insulin protecting against mitochondrial oxidative stress in septic AKI.

\section{Introduction}

Sepsis is defined as life-threatening organ dysfunction caused by a dysregulated host response to infection and is associated with high mortality and huge social burden (1). Acute kidney injury (AKI) is present in over $40 \%$ of septic patients and increases the mortality up to $70 \%(2,3)$. Mitochondria have important roles in both physiological and pathophysiological processes, including calcium homeostasis, cell signaling pathways, transcriptional regulation, and apoptosis (4). Previous studies indicated that multiple aspects of mitochondrial dysfunction contribute to septic AKI, such as the overproduction of reactive oxygen species (ROS), the depletion of adenosine triphosphate (ATP), the dissipation of mitochondrial membrane potential (MMP) and the exacerbation of apoptosis (5-8).

Oxidative stress plays an important role in the development of mitochondrial dysfunction during septic AKI. When the antioxidant protection mechanisms and ROS production are imbalanced, oxidative stress results and leads to the inactivation of endogenous antioxidant systems, disruption of the electron transport, uncoupling of mitochondrial oxidative phosphorylation, and transition of mitochondrial membrane permeability (8-10). Mitochondria is the primary source of ROS within cells (11). Therefore, mitochondrial oxidative stress-mediated damage may be the main cause of mitochondrial dysfunction.

Mitochondria-targeted antioxidants were reported to block ROS generation within mitochondria as well as preserve mitochondrial complex respiration, ATP level, and MMP both in animal and cell models of septic AKI $(9,10)$. Targeting antioxidants to mitochondria may offer a new therapy in the future but are not yet available in clinical work. Insulin therapy is a simple and inexpensive intervention for disturbed glucose and lipid metabolism during sepsis and has been applied in critically ill patients $(12,13)$. In an early experiment, we found insulin infusion increased MMP and attenuated inflammatory 
response in liver sections of septic rats (14). But the effects and mechanisms of insulin against mitochondrial oxidative stress remain to be fully elucidated. Under normal conditions, mitochondria protect from damage by ROS via several interacting antioxidant systems $(15,16)$.

We hypothesized that insulin could alleviate mitochondrial oxidative stress and aimed to explore the relations between insulin therapy and endogenous antioxidant systems as well as other mechanisms within mitochondria in a rat model of septic AKI.

\section{Materials and methods}

Animals. This study was approved by the Institutional Animal Care and Use Committee of Southern Medical University (Guangzhou, China) and conducted in accordance with the Guide for the Care and Use of Laboratory Animals of the National Institutes of Health $(\mathrm{NIH})$. Male SD rats (weighing 250 to $300 \mathrm{~g}$ ) were housed in a $25^{\circ} \mathrm{C}$ room temperature, illuminated in a $12 \mathrm{~h}$ light/12 h dark cycle with free access to water and food.

Sepsis model and drug administration methods. Rats were divided randomly into four groups ( $\mathrm{n}=6$ in each group) as follow: Control group, sham surgery group, cecal ligation and puncture (CLP) group, and CLP plus insulin group. CLP and sham surgery were performed according to the method described by Rittirsch et al (17). Before operation, all rats were anesthetized with an intraperitoneal injection of pentobarbital sodium. The rats in the CLP plus insulin group also received a subcutaneous injection of insulin glargine (Lantus ${ }^{\circledR}$; Sanofi-Aventis, Paris, France) at a dose of $0.5 \mathrm{IU} / \mathrm{kg}$ body weight. After surgery, rats were placed back in cages with free access to water and food, and the rats of CLP group were resuscitated by injecting prewarmed normal saline $\left(37^{\circ} \mathrm{C}\right.$; $5 \mathrm{ml} / 100 \mathrm{~g}$ body weight) subcutaneously. Rats were killed at 12 or $24 \mathrm{~h}$ after CLP or sham surgery as separate experiments. At sacrifice, blood specimens were obtained from abdominal aorta and renal cortices were dissected and stored at $-80^{\circ} \mathrm{C}$ until analyses.

Serum biochemical assays. The concentrations of blood urea nitrogen (BUN) and serum creatinine (CRE) were measured using an automatic biochemical analyzer (Cobas c702; Roche Diagnostics, Mannheim, Germany). The serum neutrophil gelatinase-associated lipocalin (NGAL) was estimated by a commercial ELISA kit (EK-Bioscience Biotechnology, Shanghai, China).

Histology analysis. For histopathological observation, the renal cortices were fixed, embedded, sectioned, and then subjected to hematoxylin and eosin (H\&E) staining. The images were captured by a light microscopy (Olympus BX60; Olympus America, Inc., Melville, NY, USA).

Measurement of MMP as well as levels of mitochondrial superoxide dismutase 2 (SOD2) and ROS. Renal mitochondria were isolated using the Mitochondria Isolation kit (Applygen Technologies Inc., Beijing, China). MMP was assessed with JC-1 staining and calculated as the fluorescence ratio of red (JC-1 polymer) to green (JC-1 monomer). The activity of SOD2 was evaluated using a SOD assay kit-WST method. Both above diagnosis kits were provided by Beyotime Institute of Biotechnology (Haimen, China). ROS production within mitochondria was assessed with 6-chloromethyl-2',7'-dichlorofluorescein diacetate (CM-H2DCFDA) staining (Genmed Scientifics Inc., Shanghai, China). Fluorescence or absorbance was detected by a multifunctional microplate reader (SpectraMax M5; Molecular Devices, LLC, Sunnyvale, CA, USA).

Tissue biochemical analyses. The renal cortical tissues were homogenized and the protein concentrations in the supernatant were determined using a Bradford assay kit. ATP level was detected using a firefly luciferase-based ATP assay kit. Total nitric oxide (NO) production was indicated by the concentration of nitrate and nitrite using a modified Griess reaction method. All above diagnosis kits were provided by Beyotime Institute of Biotechnology. Chemicals used for measuring the activity of inducible NO synthase (iNOS) as well as the contents of total glutathione (GSH) and oxidative GSH (GSSG) within kidney were obtained from Nanjing Jiancheng Bioengineering Institute (Nanjing, China). The production of intrarenal uncoupling protein 2 (UCP2) was estimated by a commercial ELISA kit (Oulu Biotechnology, Shanghai, China).

Immunohistochemistry. After being deparaffinized and dehydrated, the embedded kidney sections were treated with $3 \%$ hydrogen peroxide to inactivate endogenous peroxidase. Blocking buffer (10\% normal goat serum) was applied at room temperature for $30 \mathrm{~min}$ followed by sequential application of anti-UCP2 antibody (1:100; Protein Tech Group, Wuhan, China) and anti-PINK1 (1:100; Bioworld Technology, Nanjing, China), goat anti-rabbit secondary antibody and diaminobenzidine tetrahydrochloride substrate (Dako; Agilent Technologies, Inc., Santa Clara, CA, USA). The images were captured by a light microscopy (Olympus BX60; Olympus America Inc.).

$R T-q P C R$. Total RNA was extracted from the renal tissue using TRIzol reagent and then reverse transcribed and synthesized into cDNA using RT-PCR kits (Takara Biotechnology Co., Ltd., Dalian, China). RT-PCR amplification reaction was performed on the LightCycler 480 (Roche Diagnostics, Laval, $\mathrm{QC}$, Canada). The quantification cycle $(\mathrm{Cq})$ was obtained from triplicate samples and averaged. Calculations were based on the ' $\Delta \Delta \mathrm{Cq}$ method' (18) using the equation $\mathrm{R}$ (ratio) $=2^{-\Delta \Delta \mathrm{Cq}}$ and standardized by the reference gene, GAPDH. All PCR primers were provided by Generay Biotechnology (Shanghai, China). SOD1 primer: 5'-TTAGCAGGACAGCAGATGAGT-3' (forward) and 5'-TCCACGAGAAACAAGATGACT-3' (reverse). SOD2 primer: 5'-CACTACTACAAAACACCCACC-3' (forward) and 5'-CTGCTCTAATCAGGACCCACT-3' (reverse). Glutathione synthetase (GSS) primer: 5'-CTATGGCACGCTGGTCAA ATA-3' (forward) and 5'-TTCCAAGATCCTGTCCAACAA-3' (reverse). UCP2 primer: 5'-ACCATTGCACAGAGGAAGG-3' (forward) and 5'-TCTTGACCACATCAACGGG-3' (reverse). PINK1 primer: 5'-CCT TCA ACAGTTCAGGCGGT-3' (forward) and 5'-GCCTCGGTGACAGCTAAGTC. PGC1 $\alpha$ primer: 5'-GGGGCACATCTGTTCTTCCA-3' (forward) and 5'-GCTTGACTGGGATGACCGAA. GAPDH primer: 5'-GCC 
AGCCTCGTCTCATAGACA-3' (forward) and 5'-AGAGAA GGCAGCCCTGGTAAC-3' (reverse).

Blood glucose measurement. Capillary blood samples $(10 \mu \mathrm{l})$ from the tail were analyzed using a BG meter (Abbott Optimum Xceed; Abbott Diabetes Care, Inc., Alameda, CA, USA) at $0,6,12,18$ and $24 \mathrm{~h}$ time point of the experiment.

Statistical analysis. Data were expressed as mean \pm standard deviation. For multiple comparisons, we used one-way ANOVA, followed by least-significant difference tests between multiple groups. Statistical analyses were carried out using SPSS 17.0 software (SPSS, Inc., Chicago, IL, USA). $\mathrm{P}<0.05$ was considered to indicate a statistically significant difference.

\section{Results}

Insulin attenuated sepsis-induced AKI. As shown in Fig. 1A, indicators of renal injury, including BUN and serum CRE were dramatically elevated at 24-h timepoint meanwhile serum NGAL were dramatically elevated both at 12 and 24-h in the CLP group compared with control or sham surgery group (all $\mathrm{P}<0.05)$. Insulin therapy attenuated these elevations (all $\mathrm{P}<0.05)$. Kidney injury was also examined by $\mathrm{H} \& \mathrm{E}$ staining (Fig. 1B). Renal tissues of the CLP group demonstrated obvious pathological alternations, such as extensive swelling and vacuolization of tubular cells. But renal sections of rats treated with insulin showed lesser pathological changes.

Insulin preserved renal mitochondrial function in septic rats. Renal mitochondrial function was analyzed by intrarenal ATP level and MMP. Fig. 2 shows that there was a sharp decline in ATP content and MMP in the CLP group compared with control or sham surgery group (all $\mathrm{P}<0.05$ ). However, insulin therapy reversed the depletion of ATP and dissipation of MMP at $24 \mathrm{~h}$ following CLP (both $\mathrm{P}<0.01$ ).

Insulin ameliorated mitochondrial oxidative stress in septic rats. The electron transport chain is the major source of intracellular ROS and mitochondria are also capable of producing NO and other reactive nitrogen species (RNS). The overproduction of NO is normally induced by NOS in sepsis. As shown in Fig. 3, the activity of intrarenal iNOS as well as production of mitochondrial ROS and NO were dramatically elevated in the CLP group compared with control or sham surgery group (all $\mathrm{P}<0.05)$. Insulin therapy attenuated these increases at $24 \mathrm{~h}$ following CLP (all $\mathrm{P}<0.05)$.

Insulin on expression of mitochondrial SOD. Endogenous antioxidant systems lie the first line of defense against oxidative stress, since they locate near the site of mitochondrial ROS formation. Normally, ROS within mitochondria is converted to hydrogen peroxide $\left(\mathrm{H}_{2} \mathrm{O}_{2}\right)$ by the action of SOD and then removed by the oxidation of reduced GSH. As demonstrated in Fig. 4, a significant decrease in the mRNA level of SOD1 and SOD2 was both detected in the CLP group compared with control group at 24 and $12 \mathrm{~h}$ following CLP respectively (all $\mathrm{P}<0.05)$. Insulin therapy increased the mRNA level of SOD2 but not SOD1 compared with CLP group at 24-h time point $(\mathrm{P}<0.001)$. Concurrently, the activity of mitochondrial SOD2 was significantly lower in the CLP group than that of control or sham surgery group $($ all $\mathrm{P}<0.001)$ and dramatically elevated by insulin treatment at $24 \mathrm{~h}$ following CLP $(\mathrm{P}<0.001)$.

Insulin on production of intrarenal GSH. GSH is the most abundant antioxidant within mitochondria. GSS is the second enzyme in GSH biosynthesis pathway. As can be seen from Fig. 5, the mRNA level of GSS was significantly decreased in the CLP group compared with control or sham surgery group (all $\mathrm{P}<0.05$ ). However, there was no significant difference in the mRNA level of GSS between the CLP group and CLP plus insulin group at the same time point. Coupled with the transcriptional downregulation of GSS, the content of total GSH within kidney decreased in the CLP group as compared with control or sham surgery group (all $\mathrm{P}<0.001)$. Although the content of total GSH in the CLP plus insulin group was higher than that of CLP group at 12 -h time point $(\mathrm{P}<0.001)$, but the two groups had equal level at 24-h. In addition, the GSSH level was significantly elevated in the CLP group compared with control or sham surgery group at $24 \mathrm{~h}$ following CLP (both $\mathrm{P}<0.05)$ and decreased dramatically in CLP plus insulin group $(\mathrm{P}<0.05)$.

Insulin on expression of UCP2. UCPs, a mitochondrial anion carrier protein, have been found to decrease ROS emission from mitochondria via induction a proton leak across the inner membrane. UCP2 is the most wildly expressed homologue of UCPs. As illustrated in Fig. 6A, the mRNA level of UCP2 increased in the CLP group and CLP plus insulin group as compared with control group at $24 \mathrm{~h}$ following CLP (both $\mathrm{P}<0.05$ ). Fig. 6B shows a significant increase of UCP2 level in the CLP group at $12 \mathrm{~h}$ as well as in CLP plus insulin group at 12 and $24 \mathrm{~h}$ following CLP compared with control or sham surgery group (all $\mathrm{P}<0.01$ ). Moreover, the UCP2 level in the CLP plus insulin group was higher than that of CLP group at $24 \mathrm{~h}$ post CLP $(\mathrm{P}<0.01)$. Similar changes of UCP2 expression were observed by immunohistochemistry (Fig. 6C).

Insulin on expression of PINK1 protein. Accumulation of damaged mitochondria trigger selective autophagy (mitophagy) to avoid more ROS formation. Normally, mitophagy is mediated via PINK1-dependent pathway. Fig. 7A shows that the mRNA level of PINK1 upregulated in the CLP group compared with control or sham surgery group at $24 \mathrm{~h}$ following CLP (both $\mathrm{P}<0.05$ ), but CLP plus insulin group had lower mRNA levels than CLP group at 24-h time point $(\mathrm{P}<0.05)$. Similar changes of PINK1 were demonstrated by immunohistochemistry (Fig. 7B).

Insulin on the transcription of PGCl $\alpha$. Increased mitochondrial mass resulting from biogenesis can improve endogenous antioxidant system and thereby attenuate ROS production. $\mathrm{PGC} 1 \alpha$ is a major regulator of mitochondrial biogenesis. However, as shown in Fig. 8, there was no significant difference in the mRNA level of PGCl $\alpha$ among the four groups.

Blood glucose monitoring. As demonstrated in Fig. 9, compared with other three groups, blood glucose levels increased in the 


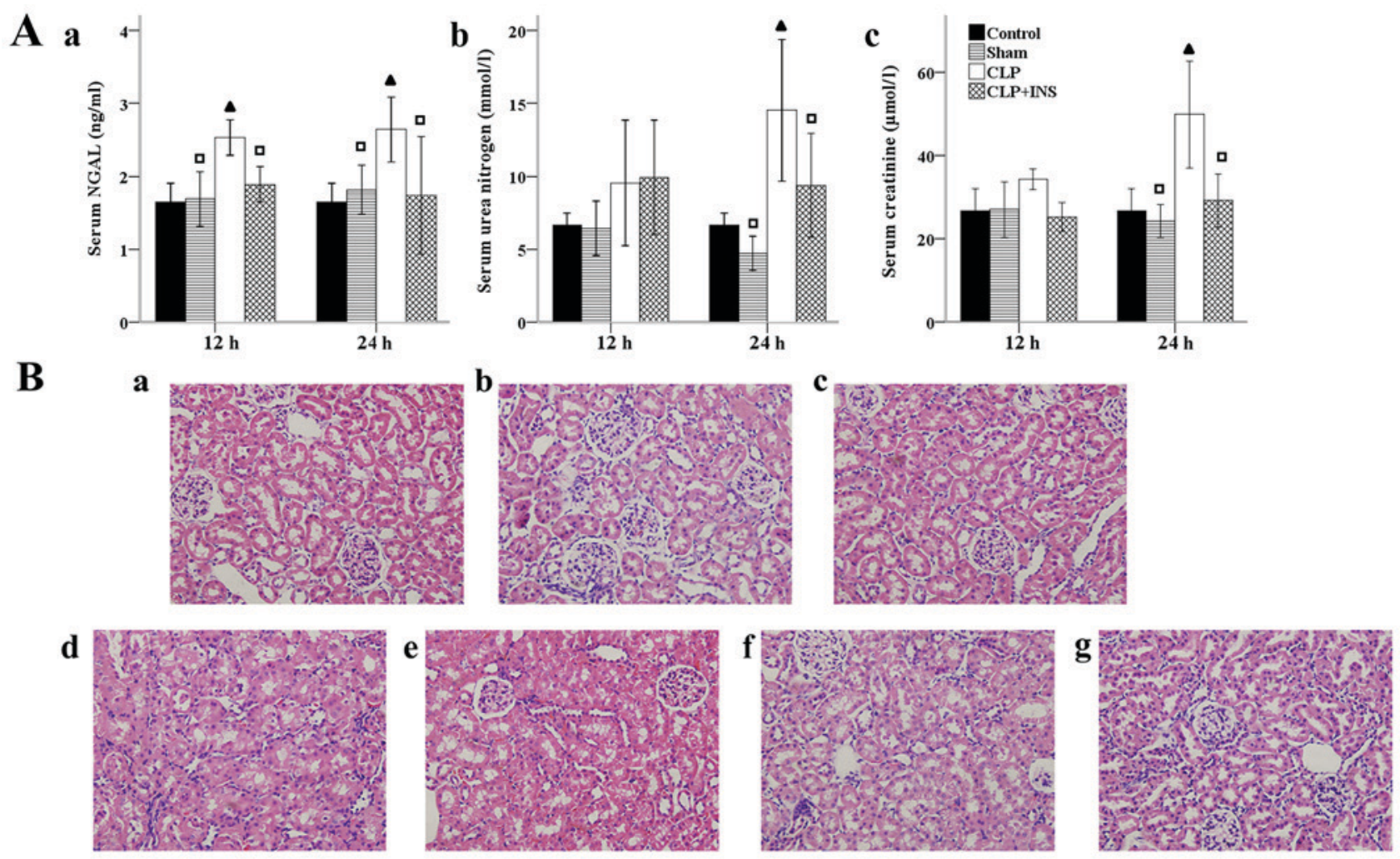

Figure 1. Effect of insulin (INS) on septic acute kidney injury. (A) Effect of INS on the levels of (a) serum neutrophil gelatinase-associated lipocalin (NGAL), (b) urea nitrogen and (c) creatinine. Results were expressed as mean $\pm \mathrm{SD}$. ${ }^{\wedge} \mathrm{P}<0.05$ vs. control, ${ }^{口} \mathrm{P}<0.05$ vs. cecal ligation and puncture (CLP) at same time point. (B) Effect of INS on sepsis-induced renal pathological changes. Panel a-g (H\&E staining, magnification, x400) represented control, sham surgery $12 \mathrm{~h}$, sham surgery $24 \mathrm{~h}$, CLP $12 \mathrm{~h}$, CLP $24 \mathrm{~h}$, CLP plus INS $12 \mathrm{~h}$, CLP plus INS $24 \mathrm{~h}$.
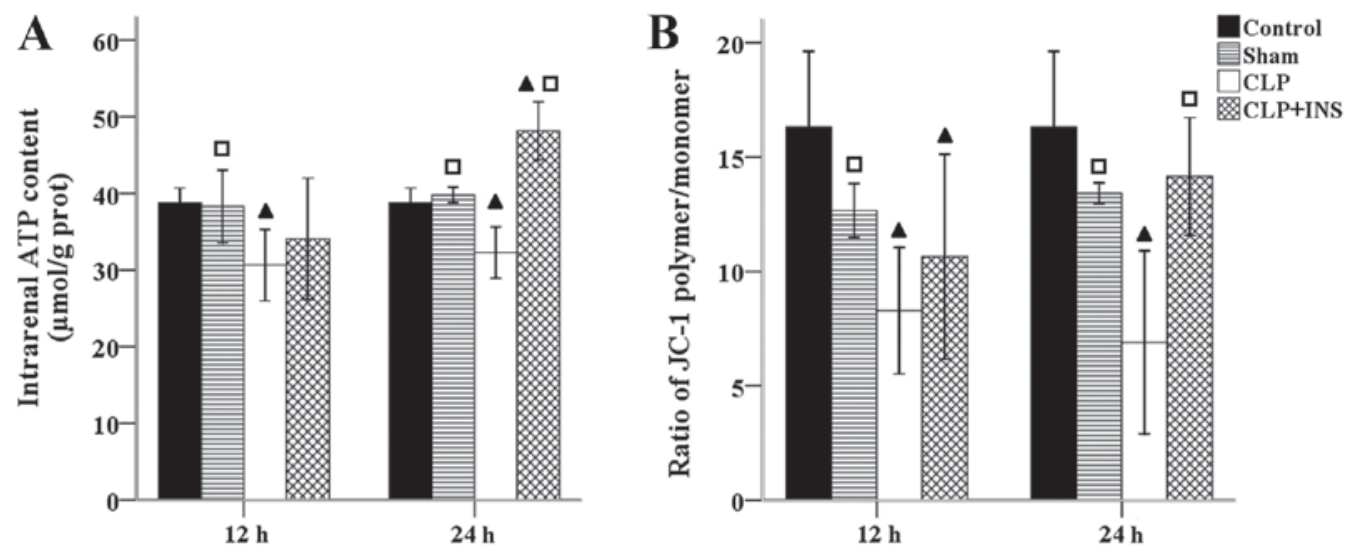

Figure 2. Effect of insulin (INS) on sepsis-induced mitochondrial injury. Levels of intrarenal (A) adenosine triphosphate (ATP) content and (B) mitochondrial membrane potential (MMP). Results were expressed as mean \pm SD. ${ }^{\wedge} \mathrm{P}<0.05$ vs. control, ${ }^{\circ} \mathrm{P}<0.05$ vs. cecal ligation and puncture (CLP) at the same time point. INS, insulin.

CLP group at 6-h time point. But the CLP group and CLP plus insulin group had equal blood glucose levels at 0,12 , 18 and $24 \mathrm{~h}$ following surgery and both lower than those of the control group or sham surgery group $(\mathrm{P}<0.05)$.

\section{Discussion}

Development of organ dysfunction under septic conditions is now accepted to result from oxidative damage to mitochondria. The present study demonstrates for the first time that insulin therapy ameliorates mitochondrial dysfunction via the suppression of mitochondrial oxidative stress involving upregulation of SOD2 and UCP2.

AKI is associated with a high morbidity and mortality in septic patients and lack of sufficient therapy $(2,3)$. The present study demonstrates that insulin treatment restored the elevations in BUN, serum CRE and NGAL following CLP. Concurrently, the histopathological alternations in renal sections were remarkably reduced in the CLP plus insulin group as compared with CLP group. The results indicate that a rat model of sepsis-induced AKI was successfully established and insulin ameliorated septic AKI. 

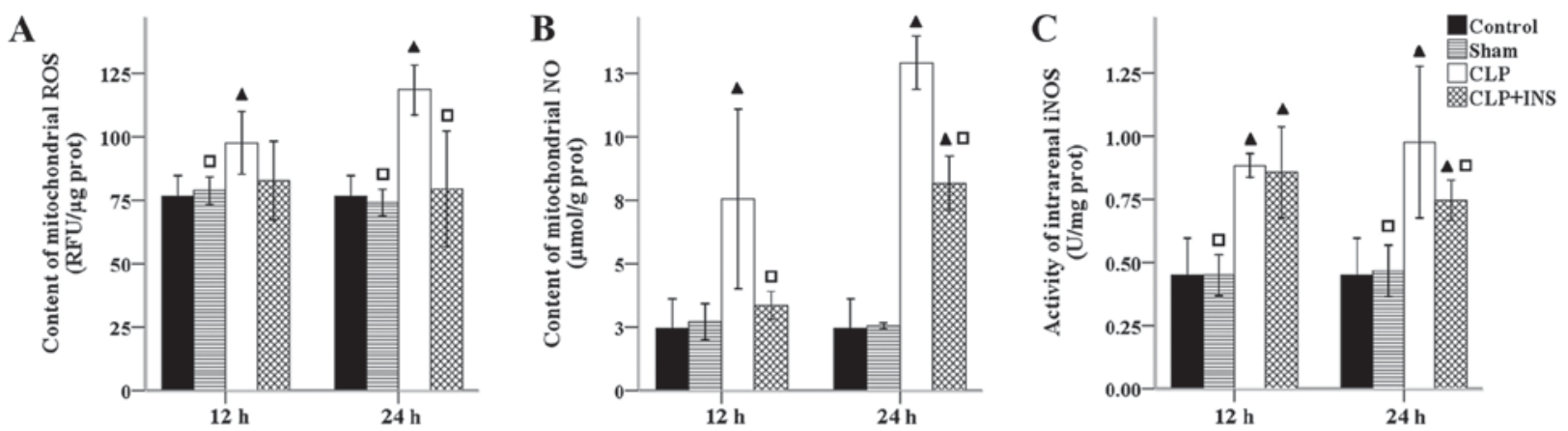

Figure 3. Effect of insulin (INS) on markers of mitochondrial oxidative stress. The contents of mitochondrial (A) reactive oxygen species (ROS) and (B) nitric oxide (NO) as the activity of (C) intrarenal inducible nitric oxide synthase (iNOS) were expressed as mean $\pm \mathrm{SD} .{ }^{\wedge} \mathrm{P}<0.05$ vs. control, ${ }^{\circ} \mathrm{P}<0.05$ vs. cecal ligation and puncture (CLP) at the same time point.
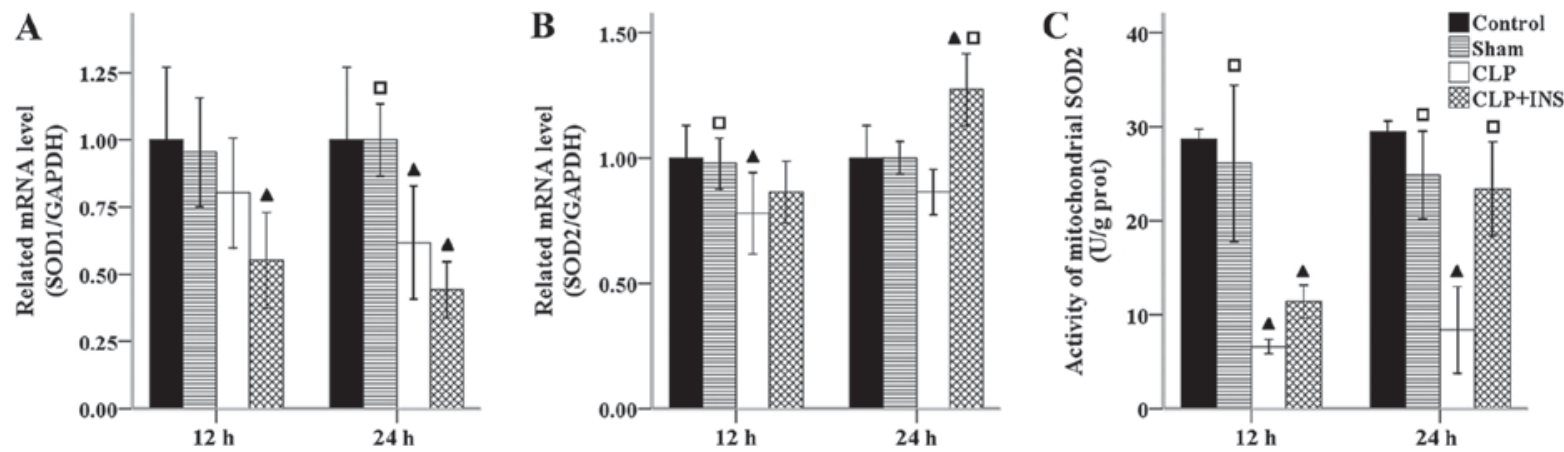

Figure 4. Effect of insulin (INS) on the expression of superoxide dismutase (SOD). The mRNA levels of (A) SOD1 and (B) SOD2 as well as the activity of (C) mitochondrial SOD2 were expressed as mean \pm SD. ${ }^{\Delta} \mathrm{P}<0.05$ vs. control, ${ }^{\circ} \mathrm{P}<0.05$ vs. cecal ligation and puncture (CLP) at the same time point.
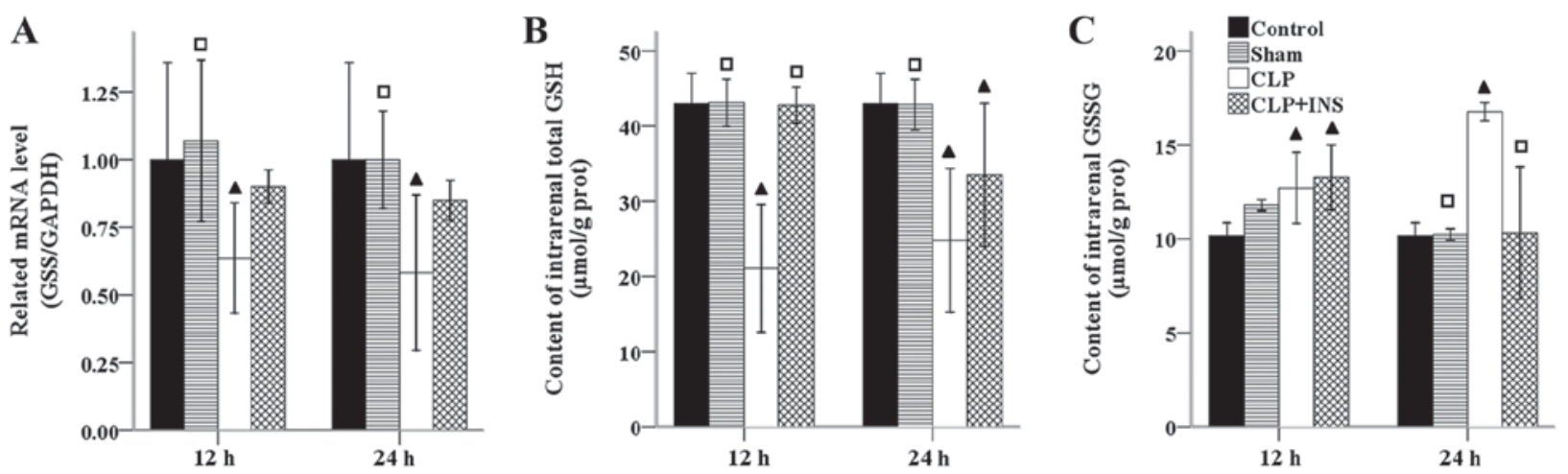

Figure 5. Effect of insulin (INS) on the expression of glutathione (GSH). The mRNA levels of (A) glutathione synthetase (GSS) as well as intrarenal content of total (B) GSH and (C) oxidative GSH (GSSG) were expressed as mean \pm SD. ${ }^{\wedge} \mathrm{P}<0.05$ vs. control, ${ }^{\circ} \mathrm{P}<0.05$ vs. cecal ligation and puncture (CLP) at the same time point.

Mitochondria influence three key processes including energy homeostasis, autophagy and cell cycle that could potentially lead to apoptosis (19). Therefore, mitochondrial dysfunction probably plays a prominent role in the development of organ dysfunction during sepsis. To our knowledge, there were no trials directly examined the protective effects of insulin on mitochondrial dysfunction in septic AKI. The present study demonstrates that insulin administration reversed the decreased levels of MMP and ATP in renal sections following CLP.

In a previous study, the group of Quoilin et al (8) has already pointed out the mechanism of ROS-induced ROS formation might be a main cause of the mitochondrial dysfunction. They revealed that after being targeted by oxidants, mitochondria became in turn producer of ROS, thus contributing to aggravate the mitochondrial dysfunction. Mitochondria is the main source and target of ROS within cells. Mitochondria-targeted antioxidants such as MnTmPyP and Mito-TEMPO have been shown to be effective in animal models of septic AKI $(9,10)$ but still not available in clinical work. Insulin administration is a simple and inexpensive therapy for disturbed glucose and lipid metabolism during sepsis and used widely in ICU $(12,13)$. Our study demonstrates for the first time that insulin lowered the production of ROS and $\mathrm{NO}$ within mitochondria following 
A

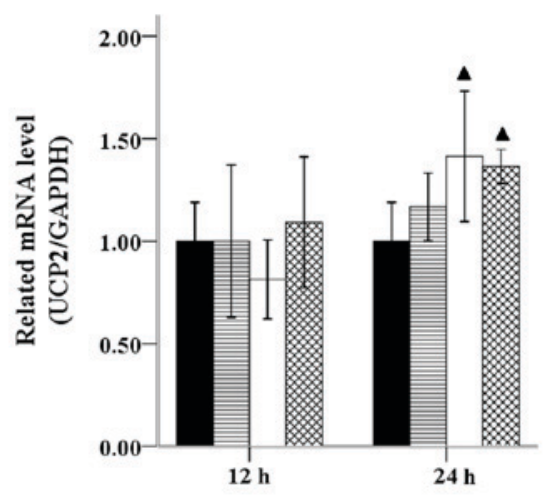

C

a

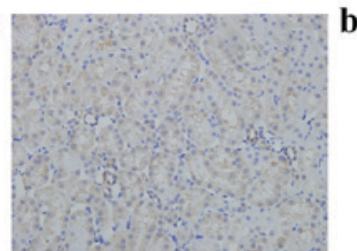

b
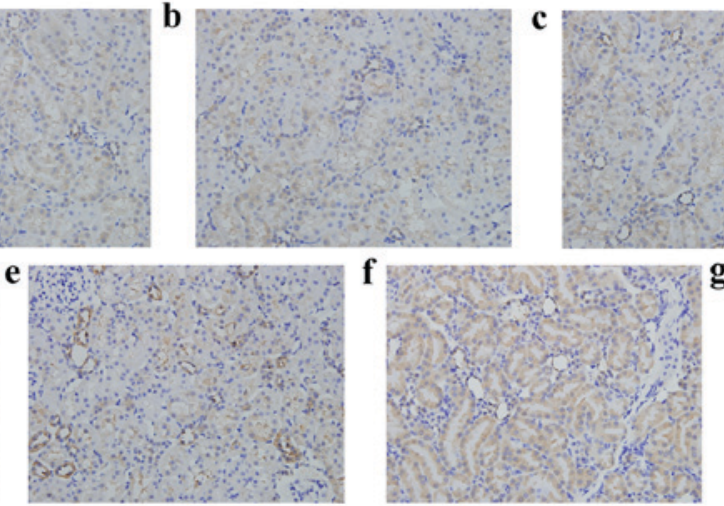

B

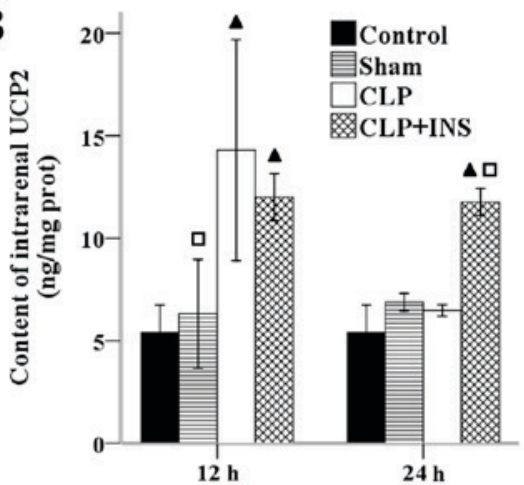

c
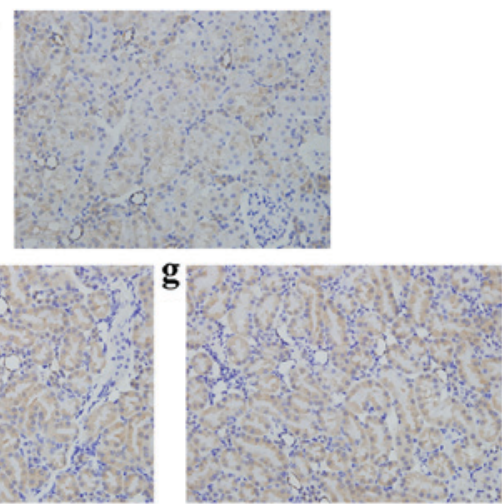

Figure 6. Effect of insulin (INS) on the expression of uncoupling protein 2 (UCP2). The mRNA levels of (A) UCP2 and the content of (B) intrarenal UCP2 were expressed as mean $\pm \mathrm{SD}$. ${ }^{\wedge} \mathrm{P}<0.05$ vs. control, ${ }^{口} \mathrm{P}<0.05$ vs. cecal ligation and puncture (CLP) at the same time point. (C) Immunohistochemical staining by using anti-UCP2 in brown. Nuclei are in blue. Panel a-g (magnification, x400) represented control, sham surgery $12 \mathrm{~h}$, sham surgery 24 h, CLP $12 \mathrm{~h}$, CLP $24 \mathrm{~h}$, CLP plus INS $12 \mathrm{~h}$, CLP plus INS $24 \mathrm{~h}$.

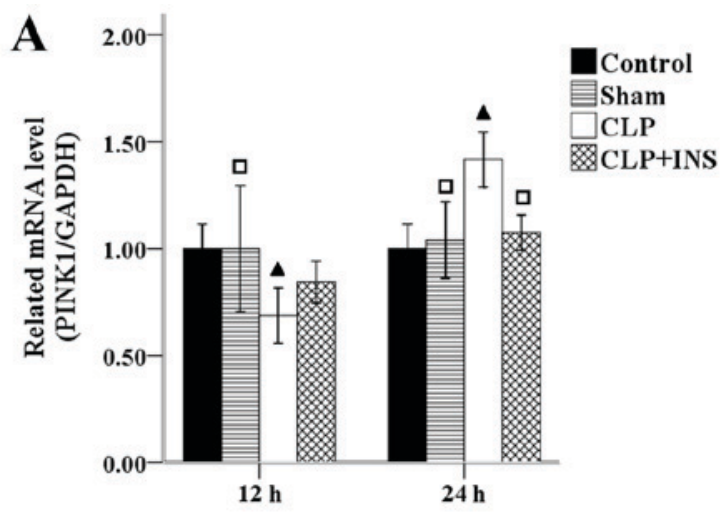

B

a

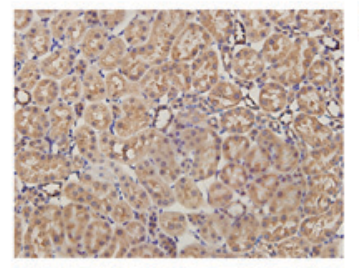

b

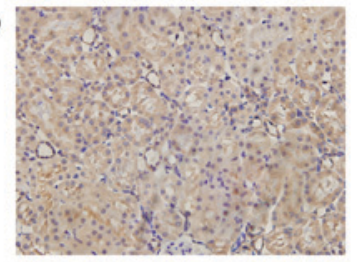

c

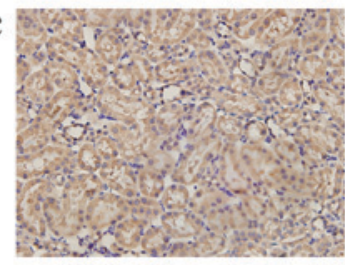

d

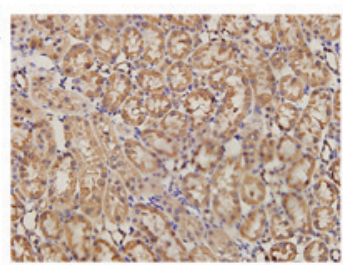

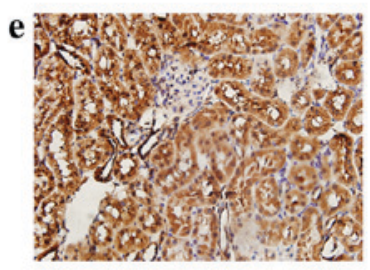

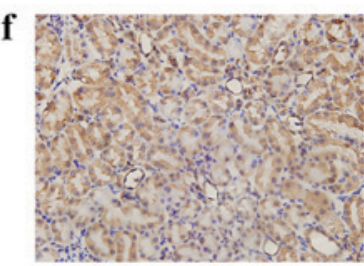

Figure 7. Effect of insulin (INS) on the expression of PINK1. The mRNA levels of (A) PINK1 were expressed as mean $\pm \mathrm{SD}$. ${ }^{\wedge} \mathrm{P}<0.05$ vs. control, ${ }^{口} \mathrm{P}<0.05$ vs. cecal ligation and puncture (CLP) at the same time point. (B) Immunohistochemical staining by using anti-PINK1 in brown. Nuclei are in blue. Panel a-g (magnification, x400) represented control, sham surgery $12 \mathrm{~h}$, sham surgery $24 \mathrm{~h}$, CLP $12 \mathrm{~h}$, CLP $24 \mathrm{~h}$, CLP plus INS $12 \mathrm{~h}$, CLP plus INS $24 \mathrm{~h}$. 


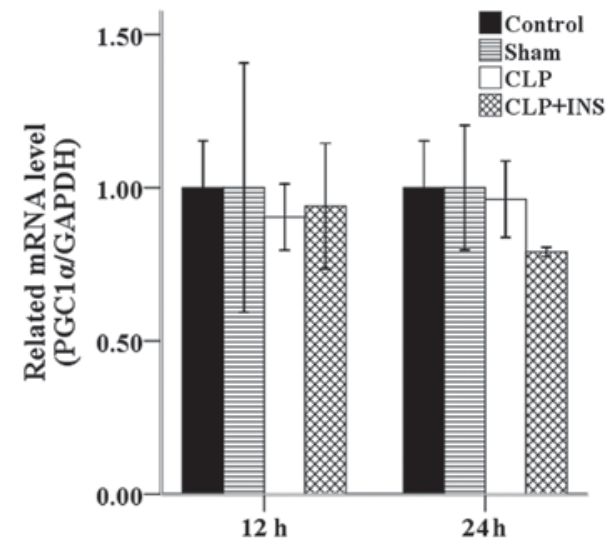

Figure 8. Effect of insulin (INS) on the mRNA levels of PGC1 $\alpha$. The results were expressed as mean $\pm \mathrm{SD}$. CLP, cecal ligation and puncture.

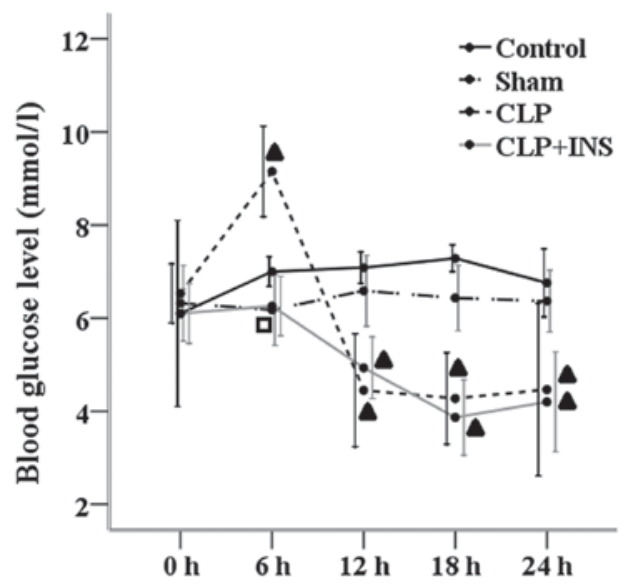

Figure 9. Blood glucose levels in the four groups. Results were expressed as mean $\pm \mathrm{SD}$. ${ }^{\Delta} \mathrm{P}<0.05$ vs. control, ${ }^{\circ} \mathrm{P}<0.05$ vs. cecal ligation and puncture (CLP) at the same time point. INS, insulin.

CLP; the deceased production of NO was due to the downregulation of iNOS. ROS cannot cross the mitochondrial membrane easily and may be more harmful than NO in oxidative damage to mitochondria (20). Our results are consistent with the observations that insulin has protective effects against the inflammatory response and oxidative stress under septic or other conditions via suppressing ROS generation and NADPH oxidase, intranuclear nuclear factor $-\kappa \mathrm{B}$ and other inflammatory mediators (21-23).

However, the precise mechanisms of insulin on mitochondrial oxidative stress remain to be fully elucidated. Hyperglycemia is common in sepsis and can also induce inflammation, oxidative stress and apoptosis in sepsis or AKI models $(24,25)$. Previous studies revealed that insulin therapy exerts the protective effect against mitochondrial oxidative stress via lowering blood glucose levels (26). However, our results demonstrate that blood glucose level do not differ in the CLP group and CLP plus insulin group except at 6-h time point, and indicate that insulin has a direct effect on mitochondrial oxidative stress rather than only an indirect via glucose modulation. Our results are consistent with the observations that insulin alleviates the inflammatory response and oxidative stress in cerebral tissues under septic conditions which was tested in a rat model (22). Several antioxidant mechanisms such as endogenous antioxidant systems, UCPs, mitophagy and mitochondrial biogenesis $(15,16)$ exist to protect against damage by ROS within mitochondria. There may be some relationship between insulin therapy and antioxidant mechanisms within mitochondria.

First of all, we investigated the modulation of insulin on endogenous antioxidant systems. Our data shows that insulin therapy upregulated the expression of SOD2 but not SOD1 in a rat model of septic AKI. In agreement with our findings, Wiryana reported that intensive insulin therapy can increase the SOD level in the ICU critically ill patients compared to conventional insulin therapy (27). ROS is usually converted into $\mathrm{H}_{2} \mathrm{O}_{2}$ by SOD2 or SOD1 located in the mitochondrial matrix and in the intermembrane space, respectively (15). SOD2 is generally thought to play a central role in the maintenance of ROS levels because it scavenges ROS at the initial step of the radical chain reaction. We propose that insulin treatment can decrease overproduction of ROS and consumption of SOD as well as upregulate the transcription of SOD2 hence leads to the increase of SOD level.

GSH is the most abundant antioxidant in mitochondria (28). The main removal of $\mathrm{H}_{2} \mathrm{O}_{2}$ is through the oxidation of reduced mitochondrial GSH into GSSH. In this study, we show that renal total GSH content in the CLP plus insulin group was higher than that of CLP group at $12 \mathrm{~h}$ following CLP, but there was no significant difference between these two groups at $24 \mathrm{~h}$. In a previous study, the group of Maitra et al has already demonstrated that rats experienced hyperglycemic phase followed by euglycemic and hypoglycemic stages during $24 \mathrm{~h}$ post CLP (29). Furthermore, Yoneyama and colleagues revealed that insulin therapy under hyperglycemia could enhance the GSH content in the liver of septic rats (24). These evidences might explain why insulin therapy failed to increase the level of GSH at $24 \mathrm{~h}$ post CLP.

UCP2 is highly expressed in kidney and normally activated directly by ROS to induce proton leak leading to membrane depolarization and ROS reduction (15). Next, we examined the alteration of UCP2 during insulin administration post CLP. To our knowledge, our study reveals for the first time that insulin upregulated UCP2 during septic AKI. Additionally, the UCP2 level in the CLP group was lower than that of CLP plus insulin group at 24-h time point which may be due to severe damage of mitochondria in the CLP group. Concurrently, we previously observed that silencing of UCP2 by small interfering RNA aggravated mitochondrial dysfunction in cardiomyocytes under septic conditions (30). Cao et al demonstrated higher level of ROS and apoptosis in UCP2-knockout mice than those of wild type (31). On the contrary, insulin has a bioactive role in the anti-oxidative and anti-apoptotic effects as well as protection of mitochondria under septic conditions $(21,32,33)$. All of these evidences may explain why UCP2 is involved in insulin ameliorating mitochondrial oxidative stress in sepsis.

In addition, our study reveals that insulin administration suppressed renal mitophagy indicated by the downregulation of PINK1 protein during sepsis. Our observation is consistent with the study of Liu et al revealed that hepatic mitophagy was suppressed in the presence of insulin resistance and 
hyperinsulinemia induced by a high fat diet in mice (34). During various conditions, the dissipation of potential across mitochondrial inner membrane leads to the redirection of PINK1 upon import and then trigger the process of mitophagy (20). We propose that insulin suppression of mitophagy may be due to the protection of mitochondrial ultrastructure. Furthermore, mitophagy requires a fully functional autophagy system, which is under stringent regulation by signaling pathways particularly mammalian target of rapamycin (mTOR) (20). As is well known, class I phosphatidylinositol-3-kinase (PI3K)-AKT activates mTOR in response to insulin signaling, acting as a negative regulator of autophagy (35). Thus, insulin itself has the property of inhibiting autophagy.

Finally, mitochondrial biogenesis was investigated by the mRNA levels of PGC1 $\alpha$ a major regulator of mitochondrial biogenesis and cellular metabolism. The fall in PGC1 $\alpha$ expression could arise as a consequence of AKI (10). Up to now, there are no trials directly the modulation of insulin on mitochondrial biogenesis in sepsis. And our data show that there was no significant difference among the four group.

In conclusion, this study demonstrates that insulin ameliorates mitochondrial oxidative stress involving upregulation of SOD2 and UCP2 in septic AKI. However, SOD pathway is an energetically costly mechanism to decrease ROS production because it is reliant on ATP and NADPH which both are decreased in sepsis (15). Furthermore, our data reveals that insulin fails to increase GSH synthesis that may impair the anti-oxidative effects of SOD. On the contrast, UCP2 was reported to accumulate rapidly in the inner mitochondrial membrane during mitochondrial reactive oxygen stress in macrophages (36). It seems that upregulation of UCP2 might be a main mechanism of insulin on mitochondrial oxidative stress under septic conditions.

\section{Acknowledgements}

Not applicable.

\section{Funding}

The present study was supported by the National Natural Science Foundation of China (grant no.81272070).

\section{Availability of data and materials}

All data generated or analyzed during this study are included in this published article.

\section{Authors' contributions}

GDC conceived the study, participated in its design, developed the study and drafted the manuscript. JLZ and YTC made significant contributions during protocol development and helped develop the study. JXZ and TW performed the sequencing results analysis, and coordinated the acquisition and interpretation of data. QYZ made significant contributions in the study design, helped interprete the data and revise the manuscript, and was responsible for the oversight of the present study.

\section{Ethics approval and consent to participate}

The present study was approved by the Institutional Animal Care and Use Committee of Southern Medical University (Guangzhou, China).

\section{Consent for publication}

Not applicable.

\section{Competing interests}

The authors declare that they have no competing interests.

\section{References}

1. Abraham E: New definitions for sepsis and septic shock: Continuing evolution but with much still to be done. JAMA 315: 757-759, 2016.

2. Bagshaw SM, George C and Bellomo R; ANZICS Database Management Committee: Early acute kidney injury and sepsis: A multicentre evaluation. Crit Care 12: R47, 2008.

3. Schrier RW and Wang W: Acute renal failure and sepsis. N Engl J Med 351: 159-169, 2004

4. Galley HF: Bench-to-bedside review: Targeting antioxidants to mitochondria in sepsis. Crit Care 14: 230, 2010.

5. Yang RL, Wang XT, Liu DW and Liu SB: Energy and oxygen metabolism disorder during septic acute kidney injury. Kidney Blood Press Res 39: 240-251, 2014.

6. Pathak E, Macmillan-Crow LA and Mayeux PR: Role of mitochondrial oxidants in an in vitro model of sepsis-induced renal injury. J Pharmacol Exp Ther 340: 192-201, 2012.

7. Lee SY, Lee YS, Choi HM, Ko YS, Lee HY, Jo SK, Cho WY and Kim HK: Distinct pathophysiologic mechanisms of septic acute kidney injury: Role of immune suppression and renal tubular cell apoptosis in murine model of septic acute kidney injury. Crit Care Med 40: 2997-3006, 2012.

8. Quoilin C, Mouithys-Mickalad A, Lècart S, Fontaine-Aupart MP and Hoebeke M: Evidence of oxidative stress and mitochondrial respiratory chain dysfunction in an in vitro model of sepsis-induced kidney injury. Biochim Biophys Acta 1837: 1790-1800, 2014.

9. Patil NK, Parajuli N, MacMillan-Crow LA and Mayeux PR: Inactivation of renal mitochondrial respiratory complexes and manganese superoxide dismutase during sepsis: Mitochondria-targeted antioxidant mitigates injury. Am J Physiol Renal Physiol 306: F734-F743, 2014.

10. Parikh SM, Yang Y, He L, Tang C, Zhan M and Dong Z: Mitochondrial function and disturbances in the septic kidney. Semin Nephrol 35: 108-119, 2015.

11. Kastl L, Sauer SW, Ruppert T, Beissbarth T, Becker MS, Suss D, Krammer PH and Gülow K: TNF- $\alpha$ mediates mitochondrial uncoupling and enhances ROS-dependent cell migration via NF- $\kappa B$ activation in liver cells. FEBS Lett 588: 175-183, 2014.

12. Mesotten D, Swinnen JV, Vanderhoydonc F, Wouters PJ and Van den Berghe G: Contribution of circulating lipids to the improved outcome of critical illness by glycemic control with intensive insulin therapy. J Clin Endocrinol Metab 89: 219-226, 2004.

13. Heuer JG, Sharma GR, Zhang T, Ding C, Bailey DL, Stephens EJ, Holmes KC, Grubbs RL, Fynboe KA, Chen YF and Jakubowski JA: Effects of hyperglycemia and insulin therapy on outcome in a hyperglycemic septic model of critical illness. J Trauma 60: 865-872, 2006.

14. Zeng QY,Zhang CM and Qian XH: Protective effects of continue insulin infusion on liver mitochondrion in the early stage of septic rats. Xi Bao Yu Fen Zi Mian Yi Xue Za Zhi 25: 525-528, 2009 (In Chinese).

15. Mailloux RJ and Harper ME: Uncoupling proteins and the control of mitochondrial reactive oxygen species production. Free Radic Biol Med 51: 1106-1115, 2011.

16. Mccreath G, Scullion MM, Lowes DA, Webster NR and Galley HF: Pharmacological activation of endogenous protective pathways against oxidative stress under conditions of sepsis. Br J Anaesth 116: 131-139, 2016. 
17. Rittirsch D, Huber-Lang MS, Flierl MA and Ward PA: Immunodesign of experimental sepsis by cecal ligation and puncture. Nat Protoc 4: 31-36, 2009.

18. Livak KJ and Schmittgen TD: Analysis of relative gene expression data using real-time quantitative PCR and the 2(-Delta Delta C(T)) method. Methods 25: 402-408, 2001.

19. Gomez H, Ince C, De Backer D, Pickkers P, Payen D, Hotchkiss J and Kellum JA: A unified theory of sepsis-induced acute kidney injury: Inflammation, microcirculatory dysfunction, bioenergetics and the tubular cell adaptation to injury. Shock 41: 3-11, 2014.

20. Raimundo N: Mitochondrial pathology: Stress signals from the energy factory. Trends Mol Med 20: 282-292, 2014.

21. Dandona P, Ghanim H, Bandyopadhyay A, Korzeniewski K, Ling Sia C, Dhindsa S and Chaudhuri A: Insulin suppresses endotoxin-induced oxidative, nitrosative, and inflammatory stress in humans. Diabetes Care 33: 2416-2423, 2010.

22. Chen Q, Yu W, Shi J, Shen J, Gao T, Zhang J, Xi F, Li J and Li N: Insulin alleviates the inflammatory response and oxidative stress injury in cerebral tissues in septic rats. J Inflamm (Lond) 11: 18, 2014.

23. Dandona P, Aljada A, Mohanty P, Ghanim H, Hamouda W, Assian E and Ahmad S: Insulin inhibits intranuclear nuclear factor kappaB and stimulates IkappaB in mononuclear cells in obese subjects: Evidence for an anti-inflammatory effect? J Clin Endocrinol Metab 86: 3257-3265, 2001.

24. Yoneyama S, Terashima H, Yamaguchi R, Tadano S and Ohkohchi N: The manner of the inflammation-boosting effect caused by acute hyperglycemia secondary to overfeeding and the effects of insulin therapy in a rat model of sepsis. J Surg Res 185: 380-387, 2013

25. Efrati S, Berman S, Hamad RA, Siman-Tov Y, Chanimov M and Weissgarten J: Hyperglycaemia emerging during general anaesthesia induces rat acute kidney injury via impaired microcirculation, augmented apoptosis and inhibited cell proliferation. Nephrology (Carlton) 17: 111-122, 2012.

26. Verbruggen SC, Joosten KF, Castillo L and van Goudoever JB: Insulin therapy in the pediatric intensive care unit. Clin Nutr 26 677-690, 2007.

27. Wiryana M: The role of intensive insulin therapy in increasing superoxide dismutase (SOD) and normalizing hyperglycemia in critically ill patients. Acta Med Indones 41: 59-65, 2009.
28. Galley HF: Oxidative stress and mitochondrial dysfunction in sepsis. Br J Anaesth 107: 57-64, 2011.

29. Maitra SR, Wojnar MM and Lang CH: Alterations in tissue glucose uptake during the hyperglycemic and hypoglycemic phases of sepsis. Shock 13: 379-385, 2000.

30. Zheng G, Lyu J, Liu S, Huang J, Liu C, Xiang D, Xie M and Zeng Q: Silencing of uncoupling protein 2 by small interfering RNA aggravates mitochondrial dysfunction in cardiomyocytes under septic conditions. Int J Mol Med 35: 1525-1536, 2015

31. Cao T, Dong Y, Tang R, Chen J, Zhang CY and Zen K: Mitochondrial uncoupling protein 2 protects splenocytes from oxidative stress-induced apoptosis during pathogen activation. Cell Immunol 286: 39-44, 2013.

32. Leffler M, Hrach T, Stuerzl M, Horch RE, Herndon DN and Jeschke MG: Insulin attenuates apoptosis and exerts anti-inflammatory effects in endotoxemic human macrophages. J Surg Res 143: 398-406, 2007.

33. Vanhorebeek I, De Vos R, Mesotten D, Wouters PJ, De Wolf-Peeters C and Van den Berghe G: Protection of hepatocyte mitochondrial ultrastructure and function by strict blood glucose control with insulin in critically ill patients. Lancet 365 : 53-59, 2005.

34. Liu HY, Han J, Cao SY, Hong T, Zhuo D, Shi J, Liu Z and Cao W: Hepatic autophagy is suppressed in the presence of insulin resistance and hyperinsulinemia: Inhibition of FoxO1-dependent expression of key autophagy genes by insulin. J Biol Chem 284: 31484-31492, 2009.

35. Choi AM, Ryter SW and Levine B: Autophagy in human health and disease. N Engl J Med 368: 651-662, 2013.

36. Giardina TM, Steer JH, Lo SZ and Joyce DA: Uncoupling protein-2 accumulates rapidly in the inner mitochondrial membrane during mitochondrial reactive oxygen stress in macrophages. Biochim Biophys Acta 1777: 118-129, 2008. 